\title{
The Prospect of Application of Extractive Reference Substance of Chinese Herbal Medicines
}

\author{
Peishan Xie ${ }^{1,2^{*}}$, Shuangcheng $\mathrm{Ma}^{3^{*}}$, Pengfei $\mathrm{Tu}^{4}$, Zhengtao Wang ${ }^{5}$, Erich Stoeger ${ }^{6}$, Daniel Bensky ${ }^{7}$ \\ ${ }^{1}$ Macau University of Science and Technology, Macau, China \\ ${ }^{2}$ Guangdong UNION Biochemical Development Co. Ltd., Guangzhou, China \\ ${ }^{3}$ National Institute for Food and Drug Control, Beijing, China \\ ${ }^{4}$ Peking University Modern Research Center for Traditional Chinese Medicine, Beijing, China \\ ${ }^{5}$ Shanghai University of Traditional Chinese Medicine, Shanghai, China \\ ${ }^{6}$ Plantasia GmbH, Oberndorf, Austria \\ ${ }^{7}$ Independent Scholar, Seattle, USA \\ Email: *psxie163@163.com, xps340112@gmail.com.com, *masc@nicpgbp.org.cn
}

Received September 21, 2013; revised October 26, 2013; accepted November 9, 2013

Copyright (C) 2013 Peishan Xie et al. This is an open access article distributed under the Creative Commons Attribution License, which permits unrestricted use, distribution, and reproduction in any medium, provided the original work is properly cited.

\begin{abstract}
The emerging development of Extractive Reference Substance (ERS) is a methodology that meets the needs for quality control for Chinese Herbal Medicines (CHM) and respects the holistic viewpoint of Traditional Chinese Medicine (TCM) and its clinical use of multiple ingredients with synergistic effects. The convention of using just a selected few Chemical Reference Substances (CRS) cannot adequately assess the quality of intact CHM. A validated chemical spectrum of an ERS provides the global characteristics in order to more specifically identify and assess targeted CHM. This paper describes the fundamental concepts, potential significance, and basic criteria of ERS, along with methods of preparation and calibration. Given the diversity of CHM, the various problems that will occur in establishing the proper process of ERS will need to be solved in a step by step manner. The ERSs of Ziziphi spinosae semen and ERS of Fritillaria thunbergii bulbus are given as examples of the development of ERS and demonstrate why we are optimistic about the utility of this approach.
\end{abstract}

Keywords: Extractive Reference Substance (ERS); Chinese Herbal Medicine (CHM); ERS R \& D Strategy; Holistic Quality Control

\section{Introduction}

The "reference substances" are indispensable substances for assessing the quality of Chinese herbal medicines (CHM) and their products. Currently, there are two kinds of available reference substances applied to Pharmacopoeia of People's Republic of China (ChP) - Herbal Reference Substance (HRS) and Chemical Reference Substance (CRS). HRS is used for microscopic and thinlayer chromatographic identification, while CRS is used for chromatographic identification and quantitative determination. Decades of practical experience have demonstrated the positive roles of HRS and CRS in routine CHM quality control; however some drawbacks have also become clear. While HRS is unequivocally necessary for microscopic identification, it is unsatisfactory for chemical identification (e.g., TLC identification) because

\footnotetext{
${ }^{*}$ Corresponding authors.
}

of the fluctuation of the chemical composition between different individual substances. As for CRS, in addition to its merits for the attribution of the corresponding chemical compounds distributed in the herbal drugs, it acts as an external standard for assay of the target component [1]. The primary issues are limited varieties, less specificity for holistic identification of the complex composition of CHM, and expense and waste of natural resources. Furthermore, as widely argued, any arbitrarily selected CRS (chemical marker) is almost irrelevant to the synergic efficacy of an individual herbal drug, much less complex formulated herbal products [2-5]. The high cost and enormous waste of resources for obtaining a single pure CRS cannot be ignored. To make one gram of the pure chemical extract often requires dozens of kilograms of the herbal drug and an enormous amount of organic solvents. The final products of CRS are so costly that it is prohibitively expensive for testing some low-cost herbal 
drugs. The higher purity is needed, the lower yield will be obtained, and the more expensive it will be for obtaining a single pure CRS. Moreover, the real signifycance of a few arbitrarily selected CRS for quality control of a multi-ingredient CHM is questionable at best. There is a consensus at present that the conventional QC approach must be changed. Recently, simultaneous determination of multiple components by HPLC has made rapid progress due to the increasingly sophisticated chromatography and $\mathrm{MS}^{\mathrm{n}}$ detection technologies, and much more information relevant to the quality has been found [6-9]. However, the aforementioned principle problems on routine quality control still remain. On the other hand, the growing QC requirement of herbal drugs is challenging the drawbacks of CRS. So the adoption of the limited extractive reference substances (ERS) ("powdered extract” in USP) by the United States Pharmacopoeia (USP) aims to coordinate expediently with the QC requirement of the corresponding Dietary supplements in the USP. The ChP has now initiated the ERS program as a candidate of reference substances in the upcoming edition. Some researchers call the ERS as "Substitute reference substance" [10]. From the perspective of researchers, the use of ERS is not just an alternative reference substance, but an advanced approach for meaningful and comprehensive quality control to match the synergistic traits of CHM. As the secondary metabolites in the herbals, the bioactive ingredients compose the chemical pattern playing the role of fingerprint for identifying each taxonomic plant species; hence regardless of what isolated chemical markers are chosen as the targets for analysis, they will likely not be relevant to the synergistic mechanisms involved in the holistic approaches of TCM [11,12]. A fullview of a chemical profile of an ERS of the given CHM through chromatographic separation may sufficiently provide the evidence to assess the samples qualitatively and semi-quantitatively (see below). Therefore, ERS can be developed as a methodology for comprehensively oriented QC reference substances. To have the ERS of CHM mature into a fully formed method of quality control and identification, the criteria for developing the ERS of CHM need to be developed in a methodical manner. This paper presents the proposal and methodologies for how this can come to fruition.

\section{Prerequisites for Establishment of ERS of CHM}

Since the 1960s, the concept and the practice of quality control of Chinese herbal medicines (CHM) in the Chinese Pharmacopoeia (ChP) has followed the model of European herbal drugs in western pharmacopoeias like the British Pharmacopoeia (BP). In the 1960's, there were no other examples on how to formulate a reasonable quality standard for herbal medicines that could serve as a precedent. While at that time the only feasible way was to emulate the quality standard monograph of chemical medicines, this concept and strategy for quality control was doomed as it was rooted in the reductionist mindset of a single-compound-oriented analysis. Common sense tells us no one chemical ingredient can be responsible for the synergic efficacy of a herbal drug. One of the traits of CHM is of diverse curative effects in the context of the composition of the various formulas. This makes it impossible to pinpoint a single specific bioactive molecule as being responsible for the efficacy of a given CHM. In recent years, along with the rapid development of multivariable analytical technologies, facilities and algorithms, the publication on simultaneous determination of multiple ingredients in CHM has exploded. The new techniques remain very expensive and will tend to exhaust the expensive and scarce chemical references substances without any more ability to truly appreciate and control the inherent quality of CHM, particularly if theses approach come into effect in routine QC. Such a prospect leads to anxiety in the herbal medicine industries although such kind of research would be welcomed in academic circles. Other open-minded herbal analysts have considered how to develop appropriate strategies that utilize multivariable analysis pragmatically in a holistic manner. It is well known that specific chemical patterns in a plant can be revealed by chromatographic analysis. The acquired chromatographic profile represents the unique character for the given species which is called "chromatographic fingerprint". The chromatographic fingerprint generated from authentic species sample can be recognized as the criterion for identification of the target species. Once the criterion is established, the chromatographic fingerprint of a standardized Extractive of the given species is being able to act as the Extractive Reference Substance (ERS). To keep the fullview of the fingerprint (peak numbers, peak-peak ratio, integrated peaks area of the column chromatography (HPLC, GC etc.) or the total color image of the Planar chromatography (HPTLC) as a whole is very effective for chemical identification and assessment of the inherent quality, particularly at the stage we are at present with a lack of sufficient knowledge about the chemical bioactivity of CHM. We cannot assume at present which peaks in the fingerprint are indispensable, which are complementary and which are inessential and can be ignored in most cases when they only exist in trace amounts. Moreover, as the understanding of how to use CHM is the result of experience accumulated over thousands of years, the holistic approach of traditional Chinese Medicine (TCM) must be respected. Given this, it is impossible to pinpoint any single molecule for being responsible for the diverse effects of a complex CHM formula, the principle way in which CHM is used TCM practitioners. Un- 
derstanding the fundamental characteristics of CHM is the cornerstone of development of ERS of CHM.

\section{ERS - The Brand-New Reference Substance in Holism Manner for Assessing the Quality of CHM}

Unlike HRS and CRS, the ERS of CHM has not yet achieved the status of a standard in spite of such limited adoption by the USP and ChP. In part, this is due to the suspicion that it is not yet truly feasible to be widely implemented. This is a normal concern at the early stages of development of any technique. There will be a process of trial and error until the method has been validated and shown to be reproducible. It will not become the panacea protocol for QA/QC of CHM. But we should take an optimistic attitude toward its research and development potential, as it will bring a new approach to the field of CHM's QC/QA: a chromatographic fingerprint as a reference that makes comprehensive quality control possible. The majority of the TCM manufacturers have always manufactured Chinese proprietary products using crude drugs as the starting materials. Given this, inconsistencies of the quality of the final products can be predicted. Over the last decade, some new emerging Chinese medicine industries have adopted new technologies and are instead using the herbal drug extracts as the starting materials. That is certainly a good beginning to improve QC/QA. Meeting the need for effective quality control of new products, ERS is also a powerful reference substance for the in-process QC. ChP get ready to launch the program of ERS of herbal medicines for upcoming edition of the Pharmacopoeia, which will undoubtedly rapidly drive forward the development of ERS.

The advantages of the ERS of CHM include:

- It can reflect the total detectable chemical characteristics of the herbal drug from a holistic view.

- It can accordingly ensure the consistent distribution of chemical ingredients batch-to-batch of the CHM products.

- The chemically attributed components in the ERS can easily be used as an available alternative for some known CRS for identification, while the intact chemical profile can provide a much more detailed quality evaluation.

- The integration value of the ERS can be used as a simple semi-quantitative analysis for quick reference.

- It is a cost/effective reference substances in quality control of CHM

\section{Basic Requirements of ERS}

Any kind of reference substance must meet the four basic universal requirements: Authenticity, Specificity, Consistency and Stability (ASCS). Focusing on the ERS, the primary requirements would be defined as follows: 1) The chromatographic fingerprint could reflect that of the original herbal drug, for example, the TLC image (fingerprint) of ERS basically conforms to that of the originnal drug. This fullfils the requirements for authenticity and specificity; 2) The physical appearance of the ERS must be robust, because most herbal drugs contain various hygroscopic components that are prone to soften and become sticky, so that the dry-powdered ERS deform. Therefore, keeping the ERS's appearance consistent is very important. The proper extraction technology should be carried out carefully to balance the diverse ingredients and principle components and be comparable with the chromatographic profiles of the original crude drugs, as well as maintaining a consistent appearance for the final ERS products. In order to fulfill the real needs, the ERS for identification and for full functionality (qualitative and quantitative) needs to take into account all four aspects of "ASCS", dealing with each aspect in turn to ensure that this project develops steadily.

Authenticity (A): Logically, the most basic source of the authenticated species of an herbal drug is from its original natural habitat. In reality, some of the so-called exemplary habitats (daodi in Chinese) have been migrating over time. For example, the exemplary habitat of the famous species of Di-Huang (Rehmannia radix) was originally in the Shanxi province of the north-west zone of China in ancient times, but it had migrated to Henan province in the Central plains zone of China by the early Ming Dynasty (1368-1644 AC). So nowadays, people know Henan province (Huaiqing county) as the exemplary habitat of Di-Huang. In the wake of increasing demand in recent decades, the area where Di-Huang is cultivated has been extended to a rather wide region. Furthermore, some Good Agriculture Practice (GAP) bases have been established beyond the original region. On the other hand, some of the original exemplary habitats of some Chinese herbal drugs have suffered environmental contamination resulting is a significant loss of quality. Additionally, as farmers have migrated to the cities, some areas that used to grow herbal drugs have been left uncultivated. Some examples of commonly-used Chinese herbal drugs that are still cultivated in the traditional exemplary habitats like Dang-Gui (Angelicae sinensis radix), Chuan-Xiong (Chuanxiong rhizoma), Bai-Zhi (Angelicae dahuricae radix), Gan-Cao (Glycyrrhizae radix et rhizoma) and Fu-Zi (Aconiti lateralis radix praeparata).

Wild-crafted Chinese herbal drugs have gradually declined due to overharvesting along with a decrease in their habitats, to the point where some have become endangered species. Therefore, we need to let go of any dogma regarding exemplary habitats given the reality of the situation today. While it is still preferable to collect samples of a given species from its traditional exemplary 
habitat if possible, often this is no longer practical and the next best approach is to obtain sufficient samples from the wholesale herbal drug markets in the main cities to ensure that they represent the main stream of the herbal distribution.

Ensuring authenticity is the first priority when dealing with the samples collected from outside the exemplary habitats. The basic testing should be carried out according to the standard in Chinese Pharmacopoeia including subjective observation of the appearance, the taste and smell, as well as chemical identification, testing and assay if necessary. Only the qualified samples can be involved in the list of candidates. It is worth noting that different samples from different habitat may have quite different chemical compositions. Sometimes, we may look at an unexpected astonishing picture in individual cases [13] (Figure 1).

Specificity (S): The ERS produced from the candidates of the given species must present a chemical ingredient pattern similar to the original crude drug in order to be considered of adequate quality. It has been well known that the most practical approach to this is to conduct chromatographic fingerprint analysis. Thin-layer chromatography method is preferred, as instant comparison is possible via the picture-like TLC image. A rough estimate can be done rapidly of the similarity among the sample images on the same plate via comparative observation of the bands numbers, band positions (Rf values), color and intensity (Figure 2); scanning profiles of the HPTLC images via corresponding digital software can be further comparative observation in detail and make more convincing assessment by similarity analysis [14] (Figures 3 and 4). HPLC fingerprint can be also applied for identification if necessary. Generally the validated chemical fingerprint of the ERS is sufficient to meet this basic requirement of an authentic herbal drug.

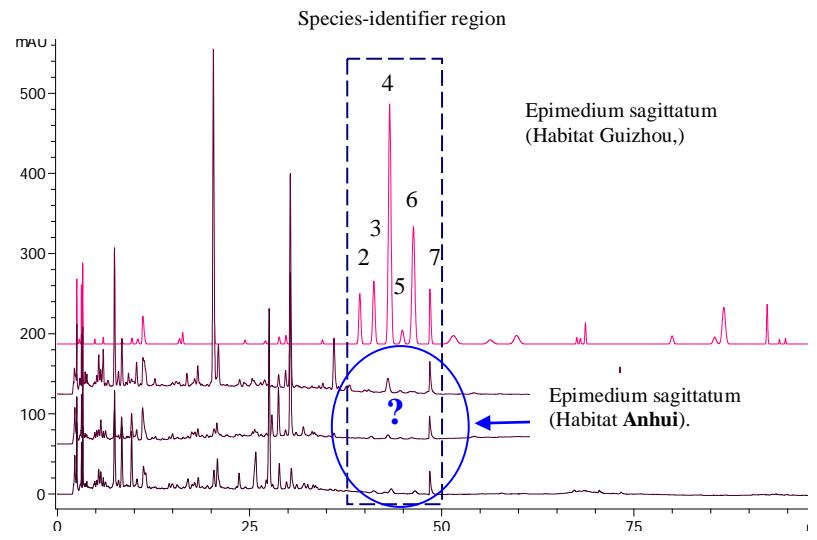

Figure 1. HPLC fingerprint of Epimedii herba (Yin-Yang-Huo) grown in different habitats. (2) epimedin A, (3) epimedin B, (4) epimedin C, (6) icariin. The three samples from the Anhui province showed none of the main bioactive flavonoids detected in "species Identifier region" of its HPLC fingerprint.

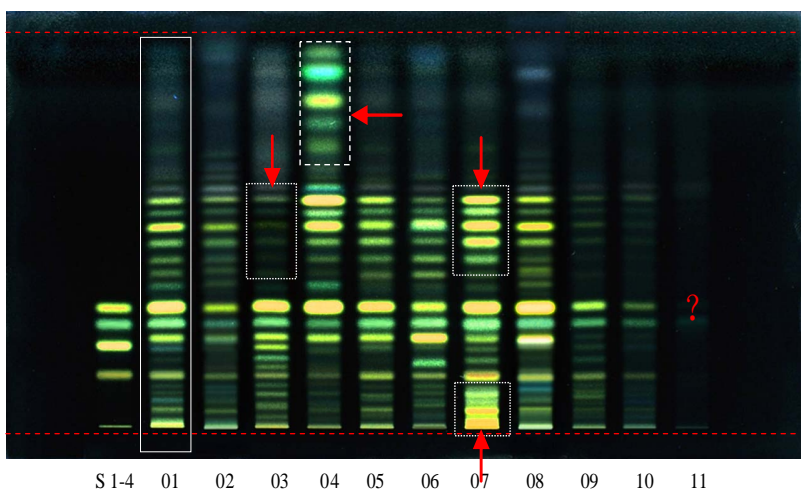

Figure 2. HPTLC fluorescence image of Bupleuri Radix (Chai-Hu). (S1): saikosaponin f; (S2) saikosaponin b2; (S3) saikosaponin a; (S4) saikosaponin d; (01) Bupleurum chinense DC.; (02) B. scorzonerifolium Willd; (03) B. longiradiatum Turcz; (04) B. bicaule Helm; (05) B. polyclonum Y. Li et S. L. Pan; (06) B. wenchuanense Shan et Y. Li (07) B. marginatum Wall. ex DC. var. stenophyllum (Wolff) Shan et Y. Li; (08) B. falcatum L; (09) B. yinchowense Shan et Y. Li; (10) B. simithii Wolff. var. parvifolia Shan et Y. Li; (11) B. tenue Huch. -Ham. ex D. Don. "Samples (01)-(09) roots; (10) (11) aerial parts.

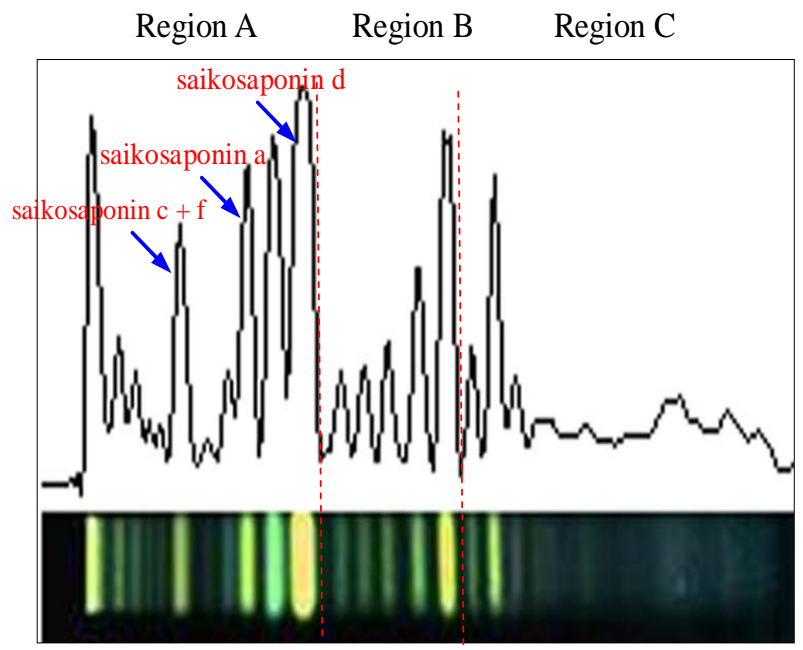

Figure 3. The digital scanning profile of the HPTLC fluorescence image of Bupleuri radix (Chai-Hu). (A): main saikosaponins region; (B): inter-species identifier region; (C) low-polarity region.

Consistency (C): A worrisome problem for ERS is inconsistencies between the chemical profiles of different batches. Therefore it is often a necessity to blend different batches of the ERS for adjustment of the ratio to reach a relatively consistent composition of the chemical ingredients based on the established common pattern of the chromatographic fingerprint and the semi-quantifiable data of the ERS.

Stability (S): The ERS must be sufficiently stable during the storage period. The tests for stability should therefore be conducted rigorously. Particularly, the phy- 


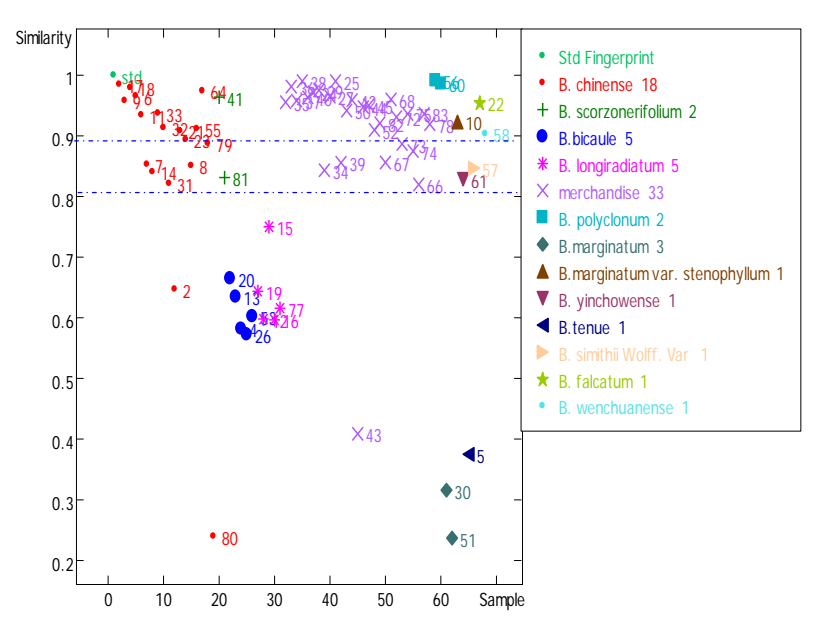

Figure 4. Similarity analysis of HPTLC fingerprint of ChaiHu.

sical appearance of the ERS must be well preserved. Generally, stickiness or softening of the appearance of ERS that occurs during storage are common problems. The solution to such problems will rely on proper extractive technologies, packaging, and storage environments. Generally, some clean-up procedures have to be done in most cases during the preparation process, so we should as far as possible balance what needs to be removed and what needs to be retained. For example, proteins, sugars, some tannins might generally be removed during the process.

In brief, the acceptable ERS should conform to the following requirements:

- A qualified ERS must be produced from authenticcated samples of the reliable habitat and include sufficient samples from various sites. Consistent raw material is necessary.

- The detected chemical composition in the ERS must be as similar as possible to that in the original herbal drug. It means that the appearance of the HPTLC image or the major composition of the HPLC profile should be fairly similar to that of original herbal drugs.

- The chemical ingredients inter-batches must be generally consistent (it works when comparing between the HPLC fingerprint or the HPTLC image). If large amount of samples need to be compared concomitantly, the similarity analysis might be conducted, then the similarity between the batches of ERS should be assumed of $>0.9$ (calculated by cosine or correlated coefficient) among batches.

- The physical state of the ERS must be stable during storage. The extractives of most CHM are quite hygroscopic. This is the major problem during storage to which particular attention must be paid during the production stage. The volatile oils in the herbal drugs will be very unstable once it is extracted by e.g., steam-distillation. Special approach for its stability need to be developed.

- A fully functional ERS requires calibration of the contents of the specified markers (cf. Section 8).

\section{Preparation of ERS of CHM}

In principle, aqueous extraction is the closest simulation of aqueous decoction practiced in TCM. However, the traditional aqueous TCM decoction contains not only water-soluble components but also some insoluble substances in suspension as the decoction is ingested without prior fine filtration. A clear aqueous solution of the herbal drugs made in the laboratory, therefore, is not equivalent to the TCM decoction. Using 60\% - 70\% methanol or ethanol extraction is the reasonable protocol to approximate the real-world decoctions. The alternative option, if necessary, is to prepare two kinds of extracts; lipophilic and hydrophilic fractions depending on the need. Some special cases with special extraction procedure should be done according to those methods that have been developed previously.

The preparation procedure of ERS includes extraction, concentration and the final formation of the ERS. In addition to the conventional extraction methods, the ecofriendly extraction methods including ultrasonic, pressurized-solvent speed extraction, smashing tissue extraction, and High-performance, High-pressure, differentially Low-temperature Successive Extraction (HHLS). This last can could be selected if the method can bear the scale of the bulk products. Some clean-up steps need to be conducted with careful and reasonable measures. No matter which method is adopted, in-process quality control must be conducted. Which method of extraction is to be selected for use will depend on the properties of the particular herb to tailor-make the ERS of targeted herbal drug which is also a matter of trial and error. One final step is the stability test. Needless to say, the storage location should have a low temperature, low humidity, and be dark. In brief, no matter which preparation method was selected, the ultimate concern would focus on whether can the final ERS products guarantee the four essential principles-Authenticity, Specificity Consistency, and Stability (ASCS).

\section{Establishing the Specification of the ERS Finished Product}

The ERS of CHM must be certified. The typical characteristics should be validated with TLC, HPLC or GC fingerprint. The standard procedure and methodology should also be validated. The characteristics of the ERS and the chromatographic fingerprint are presented together with the certification of its equivalence with the crude drug of 
the original species (raw material). The specification of ERS includes the name of the entity, the equivalent ratio of ERS/raw material, description, identification, test, assay (for full-functional ERS) and storage.

Referring to the volatile ingredients (essential oils) of the herbal drug, particularly the monoterpenes or sesquiterpenes, the chemical pattern of which are unstable, the chemical pattern would seriously fluctuate and the end product will be hard to finalize, so it is almost impossible to provide usable ERS of volatile ingredients of herbal drugs. A tentative fresh-prepared the extractive as reference by using a small sealed pouch pack of the powdered authentic raw material in reserve might be an option (Solvent extraction should be feasible).

\section{Examples of the Process and Application of ERS}

The ERS of Suan-Zao-Ren (Ziziphi spinosae semen) and Zhe-Bei-Mu (Fritillariae thunbergi bulbus) are used here as examples to demonstrate a basis for the establishment of the ERS of CHM.

\subsection{ERS of Ziziphi spinosae Semen) (ERS Suan-Zao-Ren)}

Monograph of Chinese herbs "Extractive Reference Substances” (ERS).

ERS of Ziziphi spinosae semen (defatted).

ERS of Suan-Zao-Ren (defatted)-(SZR-ERS).

Code number: SZR-RSE 2012-02 (ERS 3).

\subsubsection{Definition}

Defatted extract of Ziziphi spinosae semen for use as the Extractive Reference Substance (ERS) of Ziziphi spinosae semen. The ERS of Ziziphi spinosae semen (Chinese name: Suan-Zao-Ren) is a light grayish brown dry powder, which is $70 \%$ ethanol extraction of defatted seeds of Ziziphus jujuba Mill. var. spinosa (Bunge) Hu ex H.F. Chou (Rhamnaceae). The amount of the final product is converted to the equivalent amount of the raw material as per the extraction ratio. The ratio of the ERS to the raw material is approximately 1:14 (g, ERS/g, crude drug).

It contains spinosin 14 mg/g; jujuboside A 10.5 mg/g; jujuboside B 5.5 mg/g (Figure 5).

\subsubsection{Identification}

1) Thin-layer chromatography identification (ChP 2010 Ed) (Figure 6).

2) Criteria:

The test is not valid unless the visible color TLC image of the ERS sample shows high similarity $(>0.9$, correlation coefficient). light grayish brown to bluish green bands of jujuboside A (Rf ca. 0.16) and jujuboside B (Rf ca. 3.3) to that of the crude drug sample solution; in addition, several other weak saponin bands as well as the residual seed oil band on the front of the image also appear. Under UV $366 \mathrm{~nm}$ light, a light yellowish blue fluorescence band corresponds to spinosin CRS (Rf ca. 0.44), accompanied by one just above the spinosin band and three weak fluorescence bands lower than the spinosin band as those appeared in the crude drug sample solution.

\subsubsection{Consistency}

More than ten batches of ERS of Ziziphi spinosae semen have been checked qualitatively and quantitatively.

Carry out the method for HPLC fingerprint (optional) (Figure 7).

Assay Chromatographic Conditions: According to the method in ChP 2010 ed [6].

\subsection{ERS of Fritillariae thunbergi Bulbus) (ZBM-ERS) (ERS Zhe-Bei-Mu)}

Monograph of Chinese herbs "Extractive Reference Substances” (ERS).

ERS of Fritillariae thunbergii bulbus.

ERS of Zhe-Bei-Mu-(ZBM-ERS).

Code number: ZBM-ERS 2012-04.

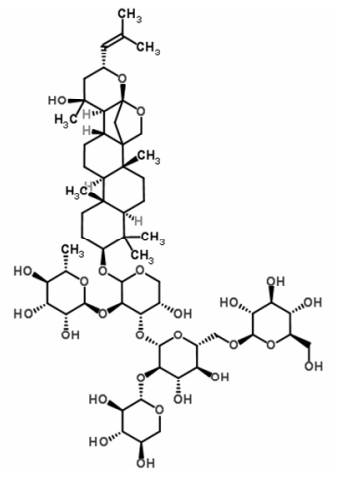

Jujuboside-a

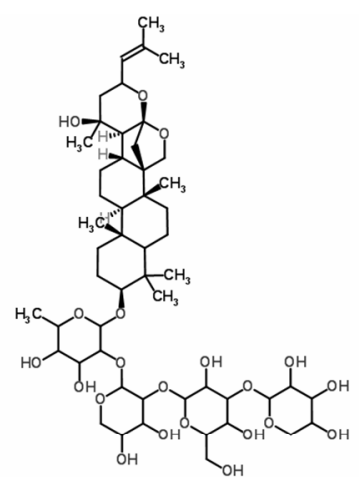

jujuboside-b

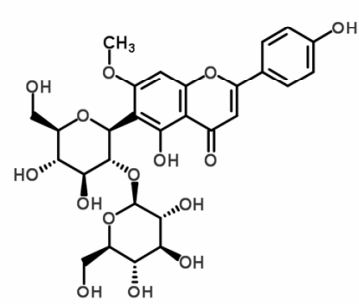

spinosin

Figure 5. The chemical structures of main saponins and flavonoid in Ziziphi spinosae semen (Suan-Zao-Ren). 


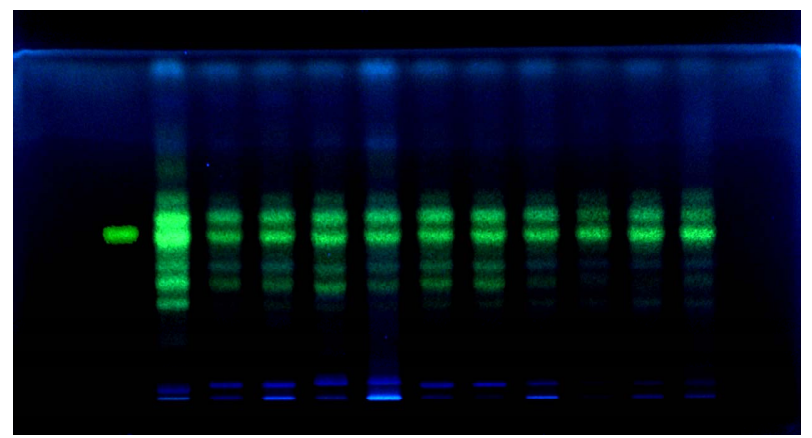

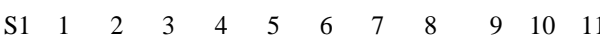

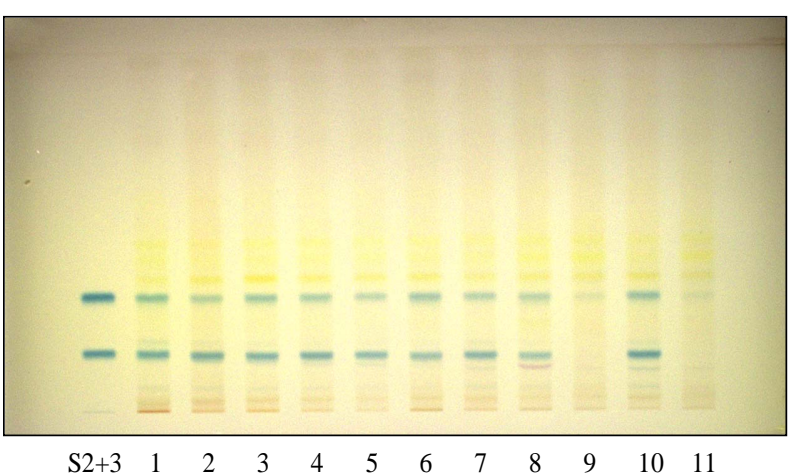

Figure 6. HPTLC images of Ziziphi spinosae semen (Suan-Zao-Ren). Above: fluorescence image of the flavonoids; Lower: visible color image of the saponin: (S1) spinosin; (S2) jujuboside A; (S3) jujuboside B; (1) ERS of defatted Ziziphi spinosae semen; (2)-(8) (10) commercial samples of Ziziphi spinosae semen; (9) (11): Adulterant (Ziziphi mauritianae semen). The HPTLC fluorescence (flavonoids) and visible color (saponin) images of the ERS of Ziziphi spinosae semen show that the authenticity and specificity of the ERS of Ziziphi spinosae semen are acceptable for a reference substance.

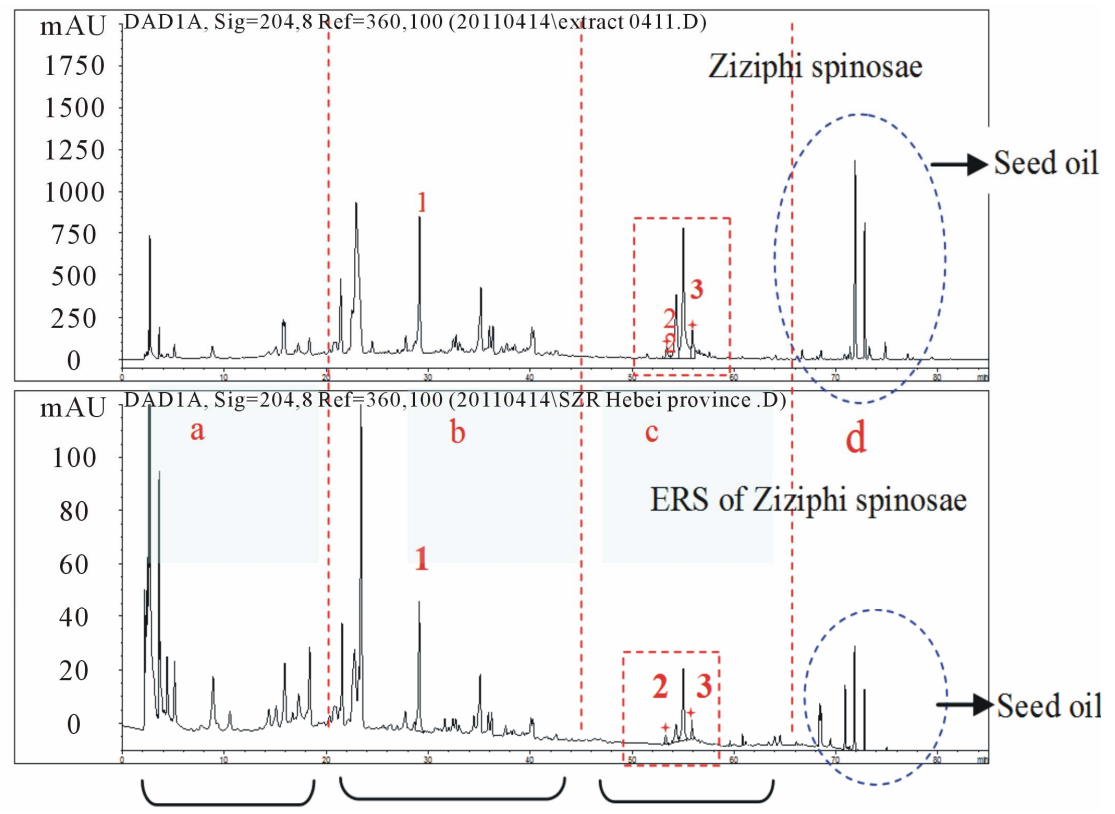

Figure 7. HPLC-DAD fingerprint of Ziziphi spinosae semen (above) and the ERS sample (lower). Peak (1): spinosin; (2): jujuboside (a); (3) jujuboside (b). The fingerprint can be divided into four sections for easy reorganization of the fingerprint's intact features. (a) alkaloids section; (b) flavonoids section; (c) saponin section; (d) seed oil section.

\subsubsection{Definition}

Extract of Fritillariae thunbergii bulbus (EFTB) for use as Extractive Reference Substance (ERS of Fritillariae thunbergii Bulbus). The EFTB is light yellowish gray dry powder, which is $70 \%$ ethanol extraction of the bulb of Fritillaria thunbergii Miq. The amount of the final product is converted to the equivalent amount of the bulb raw material as per the extraction ratio. The ratio of the ERS to the raw material is approximately 1: 10 (g/g).

It contains peimine $5.7 \mathrm{mg} / \mathrm{g}$; peiminine $5.0 \mathrm{mg} / \mathrm{g}$ (Figure 8).

\subsubsection{Identification}

Carry out the method for thin-layer chromatography
(ChP 2010 Ed.) (Figures 9 and 10).

Criteria: should demonstrate the same TLC pattern to the HRS's HPTLC image in which peimine (S1) and peiminine (S2) dominate with another light brownish orange band just above the band of peimine when sprayed with Dragendorff reagent and viewed under white light. The fluorescence image of EFTB generated by spraying $10 \%$ sulfuric acid ethanol solution observed under UV $366 \mathrm{~nm}$ is also very similar to that of the crude drug which consisted of about 10 fluorescence bands. That means the EFTB can be used as a reference substance for identification.

Confirmation: For testing the feasibility of this process, 11 batches of Fritillariae thunbergii bulbus (FTB) were 

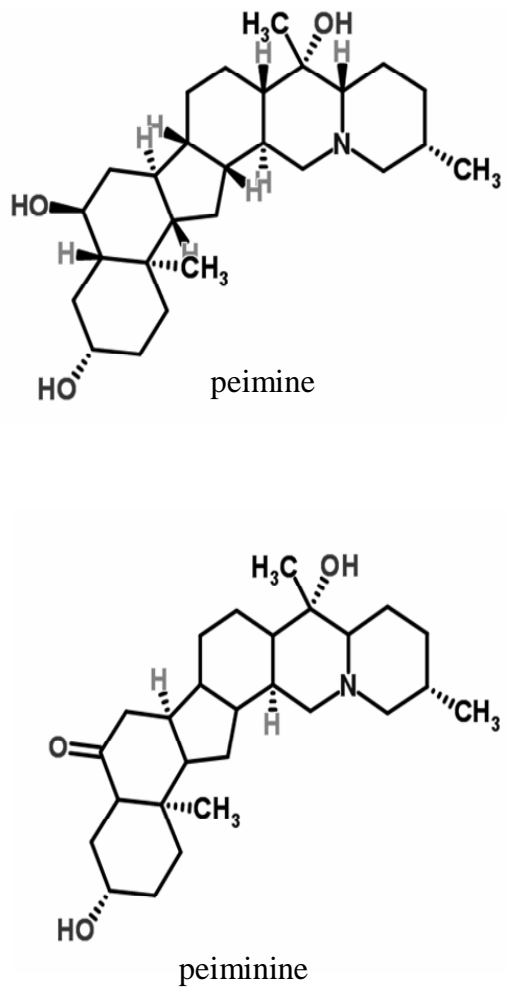

Figure 8. Chemical structure of alkaloids-peimine and peiminine.

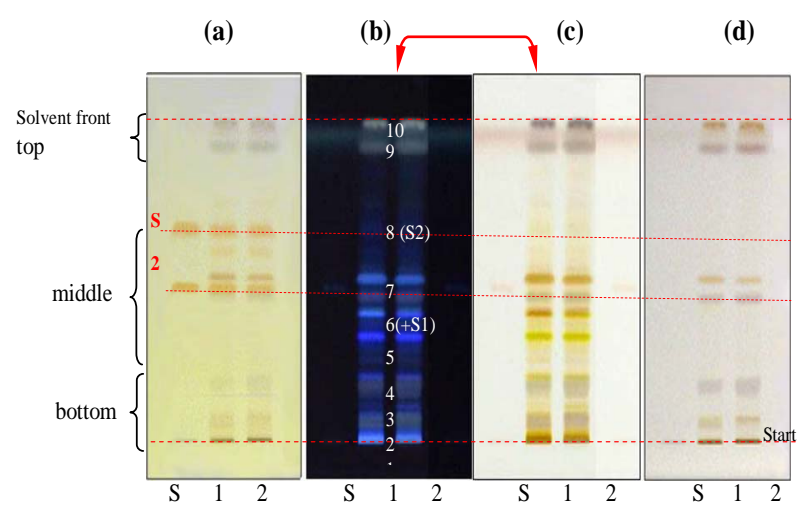

Figure 9 HPTLC images of ERS of Fritillarae thunbergii bulbus (Zhe-Bei-Mu) (1) and crude drug of Fritillarae thunbergii bulbus (2). (a): visualized by spraying Dragendorff reagent, (b): fluorescence under UV $365 \mathrm{~nm}$ after spraying sulfuric acid reagent, (c): the invert color image of (b); (d): visible image generated by spraying sulfuric acid reagent under white light. The main alkaloids-peimine (S1 $\approx$ band $6^{*}, \mathrm{Rf}$ ca. 0.46 ; and peiminine (S2 = band 8, Rf ca.0.65) stained brown bands with Dragendorff reagent. * The location of Peimine is very near to band 6 or they even overlap. The intact Fluorescence image consisted of mainly 10 blue or grayish blue fluorescence bands combined with the visible alkaloids image (a) constructed the characteristic fingerprint of FTB, the ERS of FTB (EFTB) provided very similar image with the crude drug. The legible invert color image (c) transformed from the fluorescence image (b) aided for more distinct observation.
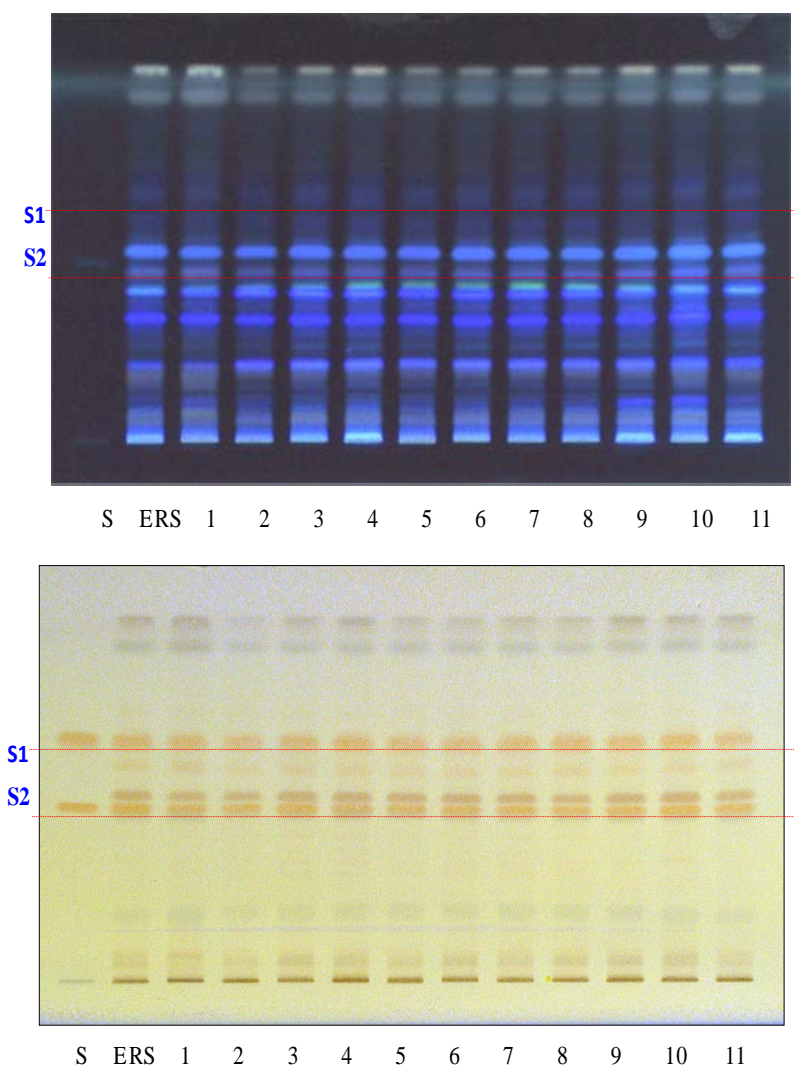

Figure 10. Application of ERS of Fritillariae thunbergii bulbus to 10 batches of Chinese herbal medicine-crude drug of Fritillariae thunbergii bulbus (FTB) T: $21^{\circ} \mathrm{C}$ RH: $50 \%$. (a)Fluorescence image after spraying $10 \%$ sulfuric acid/ethanol solution; (b) Visible color image of plate after spraying Dragendorff's reagent. $(\mathrm{S} 1)=$ peimine; $(\mathrm{S} 2)=$ peiminine; Track (1) ERS of FTB; (2)-(11) commercial crude drug-FTB The images show that the authenticity and specificity of the ERS of FTB are acceptable for a reference substance.

analyzed with the EFTB on the same plate, sample preparation and application was carried out quantitatively; the result showed the pattern of EFTB's image was basically as same as that of the crude drug FTB.

HPLC fingerprint of ERS of FTB provided a rather simple profile. As the main aklaloids, peimine and peiminine were clearly separated. It can be quantitafied by external standard method for determining their contents (Figure 11).

\section{Quantification Issue of ERS}

The use of herbal reference extractive (=ERS) instead of pure reference substance for quantitative analysis is controversial. The issue in question is the nonequivalence between the herbal extractive reference and the corresponding pure substance due to the uncertain assigned value of the analyte, chromatographic resolution and stability [15]. Most analysts have also taken for granted 


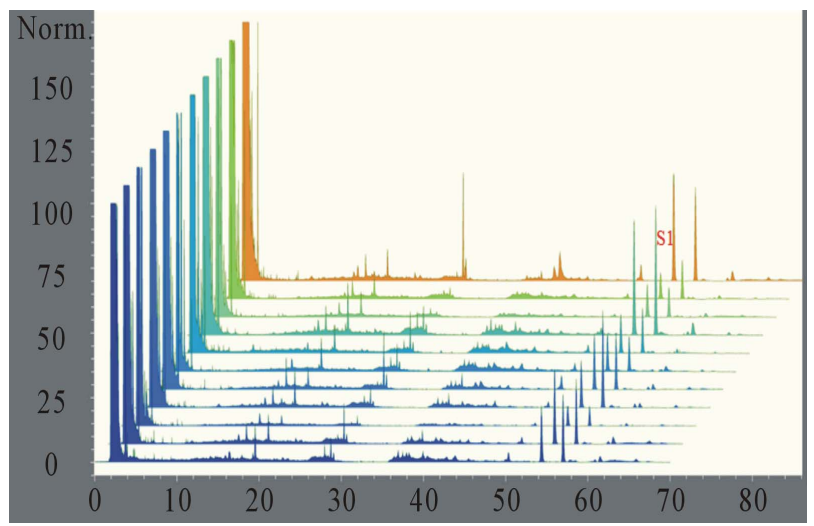

Figure 11. HPLC fingerprint of 11 batches of FTB express high similarity (cosine $>0.9$ ) compared with ERS of FTB (S1: peimine, S2: peiminine).

that one should determine a single chemical marker precisely and accurately in a herbal drug as one does the chemical pharmaceuticals. This would be true if ERS for herbal quality control was synonymous with the CRS for QC of pure chemical medicine or natural single component product. However, the situation of CHM is completely different. Determining a chemical marker for the purpose of use in TCM has to take into consideration the fact that any chemical marker is just a few parts per thousand or even a few parts per ten thousand, when the intake dose of a prescribed herbal medicine is generally 10 - $30 \mathrm{~g}$, even bigger, per potion as part of a compound formula that commonly uses $6-12$ ingredients. This situation is obviously completely different than those that inolve pure chemical pharmaceuticals. In other words, it makes no sense to treat the herbal drug exactly the same as a chemical medicine [16]. This brings us to a fundamental question: what is the real important of determining accurately and precisely such a minute amount of one or few chemical marker(s) in a herbal medicine (except for toxic ingredients)?

We suggest that it is necessary to work out the appropriate and meaningful quantitative measurements in regards to Chinese herbal medicines. In fact, all accepted external standard quantitative determinations rely on the chromatographic integrated raw data (peak area). Why not use the integrated peak area under quantitative operation conditions to rapidly estimate the semi-quantity of the contents of the all peaks or appointed peaks at the same time as performing the chromatographic fingerprint? This would be an easy, rapid, and economical approach. The raw data is rough but practically reliable and useful. One example of its utility is demonstrated by the possibility of semi-quantitative estimation of the bioactive flavonoids in Epimedium leaves through chromatographic integrated data. It is well known that the C-8-prenylated flavonoids are special bioactive contents in Epimedium spp. with the main ones being epimedin A, epimedin B, epimedin $\mathrm{C}$ and Icariin, (ABCI). A set of integrative peaks area of ABCI acquired from HPLC fingerprint demonstrated the ratio of ABCI peaks being concordant with the precisely determined content by external standard assay [13] (Figure 12). Other examples-Coptidis Rhizoma and Ginseng Radix are shown herewith the same expected results (Figures 13 and 14). The only issue with this is how to set the measurement unit to make it generally acceptable. Furthermore, it would be possible for the quantitative determination of some appointed peaks by means of calibrated ERS, in which some known chemical components were determined by external standards [10]. Of course, a good resolution of the target peak and good reproducibility is the prerequisite for this method's utility. There is an example for comparison of the quantitative determinations between using CRS and ERS to determine the contents of spinosin, jujuboside A and jujuboside B in Ziziphi spinosae semen (Suan-Zao-Ren). The primary results seem acceptable in terms of herbal medicines (Table 1).

On the quantitative analysis, Helliwell practically suggested that the key consideration on the quantification by using Herbal Drug Preparation Reference Standard ( $\approx$ RES), the content of specified constituent(s) is not an absolute value, but an assigned value determined by a specified method [17]. Our testing results by using the aforementioned method exemplified the feasibility of such a suggestion.

Our argument is that application of ERS is a more meaningful strategy for identification and semi-quantification for herbal drug quality assessment. It is true that there is still work to be done to improve the preparation and application of ERS to QA/QC for chromatographic identification and rapid quantifiable estimation and see how it can be performed in a cost-effective way. There are of course other problems that will need to be solved through trial and error, so that it is necessary to go forward one step at a time. As the saying goes that "the perfect can be the enemy of the good enough”.

\section{Discussion}

The ERS of CHM is a new methodology as well as a subject of much debate. The first issue might be what the criterion is for pragmatic useful ERS. The essential feature of the qualified ERS for identification is that it should be able to represent consistently the detected intact characteristics of the original crude drug expressed by the chromatographic fingerprint. The chemical attribution of the elements in the profile can be pinpointed by advanced technologies combined with available CRS. Considering the variety and complexity of CHM, it would be unwarranted hurdle at the early stage if over- 

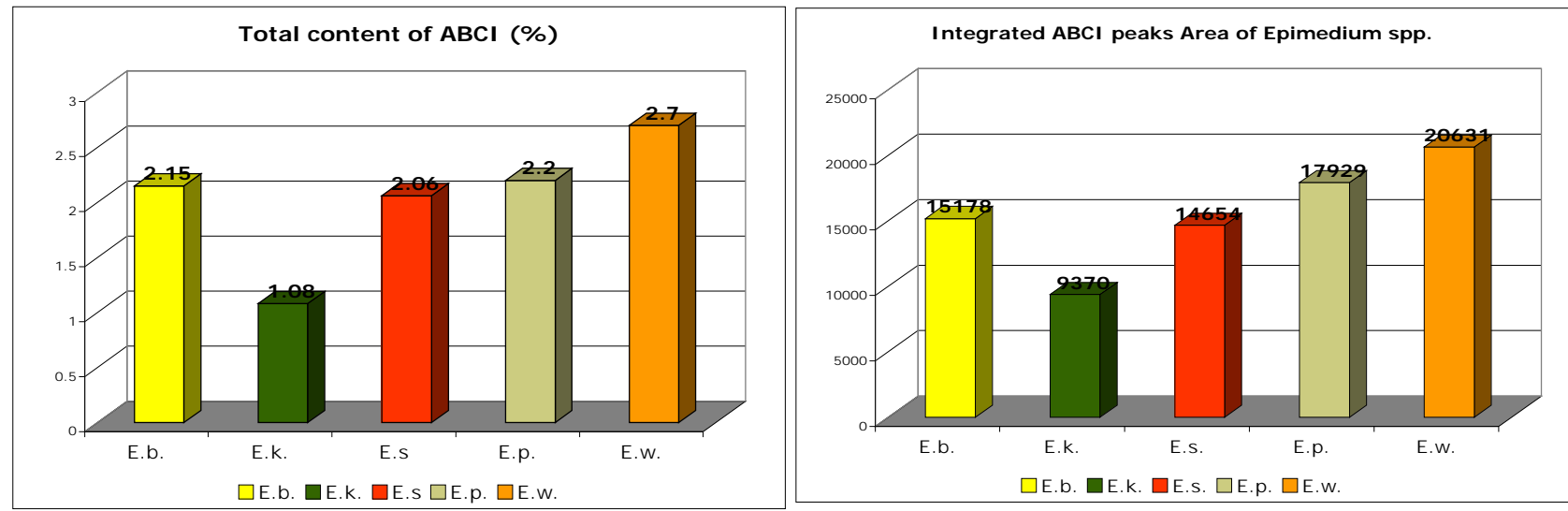

Figure 12. The comparative results of the contents of the total four C-8-prenylated flavonoids (ABCI) in epimedi herba. Light: External standard method; Right: the HPLC integrated peaks area.
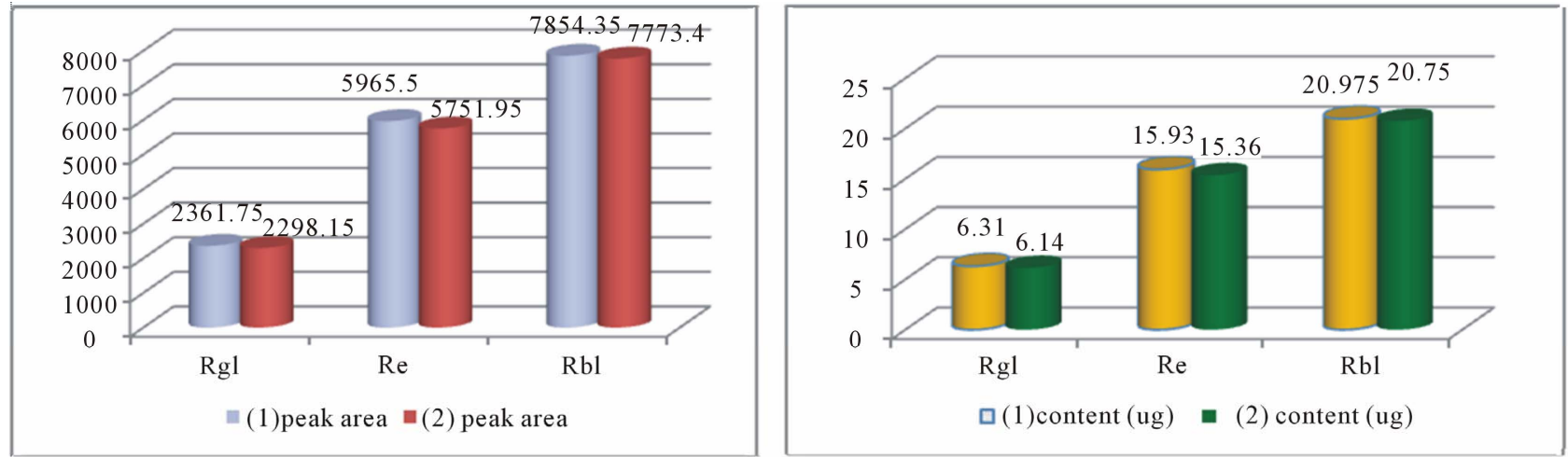

Figure 13. Comparison between integrated raw data (peak area) and external standard determination for assessing the quality of main ginsenosids in Extractive Ginseng.
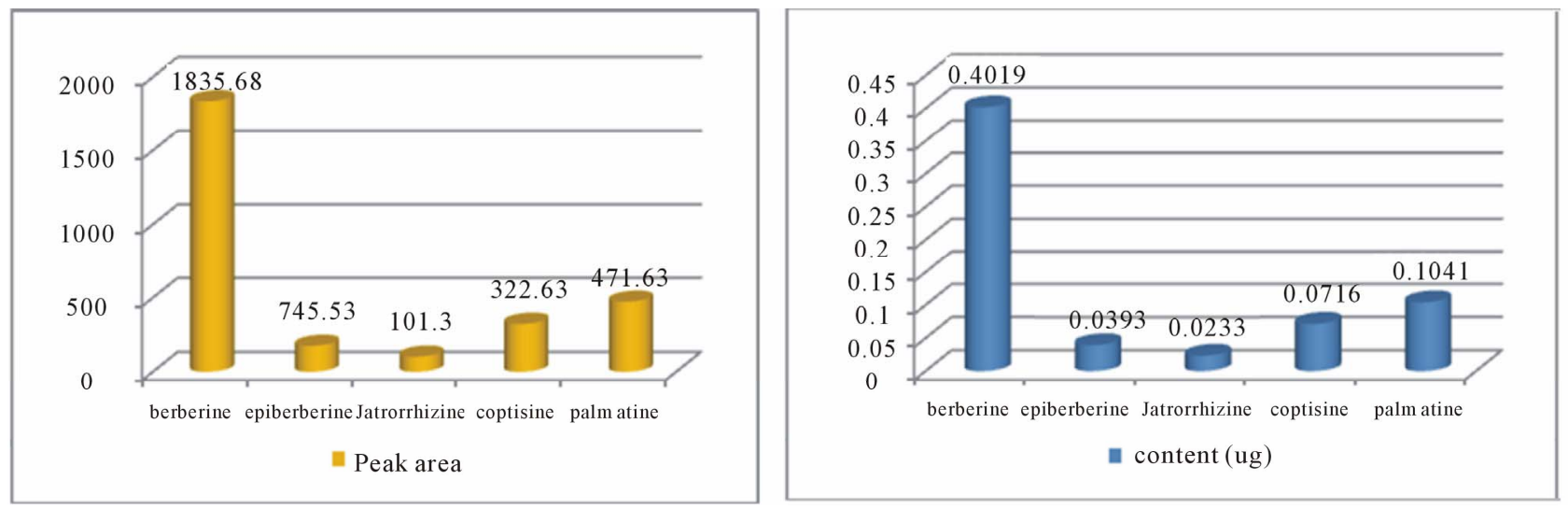

Figure 14. Comparison of the quantitative assessing between integrated raw data (peak area) and external standard method of five alkaloids in Extract of Coptidis. A rapid estimation of the contents of the five alkaloids, reading HPLC peak area value is comparable with the conventional external standard determination.

emphasizing the desire of chemical attribution in an ERS; successive exploration requires sustainable research. To be useful ERS will need to be done in a flexible manner so that it can be tailored to different situations for various types of samples. A "one-size fits all” would be impractical. The practical, economical, and relatively easily accomplished nature of ERS is a factor pushing this approach forward, particularly for the commercial and industrial field of CHM. We need to keep in mind that the conceptual integrity of the medicine and the fuzziness of many problems will direct the research and application of ERS works in concert with effective quality control of 
Table 1. Table type styles (Table caption is indispensable).

\begin{tabular}{cccc}
\hline & \multicolumn{3}{c}{ the content (\% crude drug) a } \\
\cline { 2 - 4 } & By CRS & By ERS & RSD \\
\hline spinosin & 0.0632 & 0.0634 & 0.16 \\
Jujuboside A & 0.0523 & 0.0531 & 0.76 \\
Jujuboside B & 0.0268 & 0.0287 & 3.47 \\
\hline
\end{tabular}

${ }^{\mathrm{a}}$ Using the routine external standard determination method.

CHM. Preparing a qualified practical and economical ERS of CHM requires a certain amount of expertise and diligence. Referring to the full-functional ERS, a rapid quantifiable estimation of the bioactive fraction of the whole using acquired integration data from the chromatographic fingerprint is a practical issue. Calibrating the appointed target peaks in the ERS to serve as CRS to determine the sample tested is worth investigating.

\section{Conclusion}

There is a fundamental difference in outlook between the antagonistic-oriented approach of single chemical pharmaceuticals and the orientation of TCM towards balancing the human body's function. Strategies of meaningful quality control need to take into account the complexity of CHM. No work into the safety and efficacy can afford to ignore the synergic action exerted by multi-ingredients in the herbals according to TCM constructs. Research and application of ERS, a standardized extractive with its detectable chemical pattern for a given species, are becoming a new trend for reference substances used for herbal medicine quality control. The criteria on the ERS of CHM should pursue the Authenticity, Specificity, Consistency and Stability (ASCS) in a holistic manner. Overcoming the inertia generated by dogma needs to be done as soon as possible to achieve real meaningful quality control of Chinese herbal medicines.

\section{Acknowledgements}

We appreciate our team members, Shuai Sun, Li Shao, He Li, Ruiyin Wang, Longgang Guo, Tao Kang and Xiaofeng Li, for their participation in the experiments on preparation and quality analysis of ERS cited in this paper. We also thank David Glyn Pinder for his editing assistance.

\section{REFERENCES}

[1] S. L. Li, Q. B. Han, C. F. Qiao, J. Z. Song, C. L. Cheng and H. X. Xu, "Chemical Markers for the Quality Control of Herbal Medicines: An Overview," Chinese Medicine, Vol. 3, No. 7, 2008, pp. 1-16.

[2] A. Y. Leung, “Tradition- and Science-Based Quality Control of Chinese Medicines-Introducing the PhytoTrue System,” Journal of AOAC International, Vol. 93,
No. 5, 2010, pp. 1355-1366.

[3] P.-S. Xie and A. Y. Leung, "Understanding the Traditional Aspect of Chinese Medicine in Order to Achieve Meaningful Quality Control of Chinese Materia Medica,” Journal of Chromatography A, Vol. 1216, No. 11, 2009, pp. 1933-1940.

http://dx.doi.org/10.1016/j.chroma.2008.08.045

[4] P. S. Xie, "The Basic Requirement for Modernization of Chinese Herbal Medicine, Ping-Chung Leung, Annals of Traditional Chinese Medicine, Current Review of Chinese Medicine-Quality Control of Herbs and Herbal Material, 2,” Chapter 1, 2006, pp. 1-10.

[5] S. S. Chitlange, "Chromatographic Fingerprint Analysis for Herbal Medicines: A Quality Control Tool," Pharmainfo.net, 2008. http://www.pharmainfo.net/

[6] S. L. Li, Y. X. Wang, L. H. Sheng and L. Yi, "Quality Evaluation of Radix Astragali through a Simultaneous Determination of Six Major Active Isoflavonoids and Four Main Saponins by High-Performance Liquid Chromatography Coupled with Diode Array and Evaporative Light Scattering Detectors," JCA, Vol. 1134, No. 1-2, 2006, pp. 162-169.

http://dx.doi.org/10.1016/j.chroma.2006.08.085

[7] X. J. Chen, H. Ji, Q. W. Zhang, P. F. Tu, Y. T. Wang, B. L. Guo and S. P. Li, "A Rapid Method for Simultaneous Determination of 15 Flavonoids in Epimedium Using Pressurized Liquid Extraction and Ultra-Performance Liquid Chromatography,” JPBA, Vol. 46, No. 2, 2008, pp. 226-235.

[8] C. L. Fan, J. W. Deng, Y. Y. Yang, J. S. Liu, Y. Wang, X. Q. Zhang, K. C. Fai, Q. W. Zhang and W. C. Ye, "MultiIngredients Determination and Fingerprint Analysis of Leaves From Ilex Latifolia Using Ultra-Performance Liquid Chromatography Coupled with Quadrapole Time-of -Flight Mass Sepctrometry,” JPBA, Vol. 84, 2013, pp. 2029.

[9] S. P. Li, C. M. Lai, Y. X. Gong, K. K. W. Kan, T. T. X. Dong, K. W. K. Tsim and Y. T. Wang, "Simultaneous Determination of Ergosterol, Nucleosides and Their Bases from Natural and Cultured Cordyceps by Pressurised Liquid Extraction and High-Performance Liquid Chromatography,” JCA, Vol. 1036, No. 2, 2004, pp. 239-243.

[10] P. Yu, S. Lei, H. Y. Jing and S. C. Ma, "Discussion on Application and Technical Requirements of Substitute Refeemce Substance Method for Simultaneous Determination of Multi-Components in Traditional Chinese Medicine,” Chin. J. Pharm. Anal, Vol. 33, No. 1, 2013, pp. 169-177.

[11] J. S. Zhang and C. H. Lu, "Philisophical Origins of Source of Traditional Medicine's Holism and Western Medicine's Reductionism," Journal of Anhui Traditioanal Chinese Medicine College (in Chinese), Vol. 18, No. 1, 1999, pp. 1-3.

[12] X. D. Tang and W. W. Wang, "TCM Research: Cultural Collision and System Biology," World Science and Technology/Modernization of Traditionl Chinese Medicine and Materia Medica (in Chinese), Vol. 9, No. 1, 2007, pp. 119-122.

[13] P. S. Xie, Y.-Z. Yan, B.-L. Guo, C. W. K. Lam, S. H. 
Chui and Q.-X. Yu, “Chemical Pattern-Aided Classification to Simplify the Intricacy of Morphological Taxonomy of Epimedium Species Using Chromatographic Fingerprinting," Journal of Pharmaceutical and Biomedical Analysis, Vol. 52, No. 4, 2010, pp. 452-460.

http://dx.doi.org/10.1016/j.jpba.2010.01.025

[14] R.-T. Tian, P.-S. Xie and H.-P. Liu, "Evaluation of Traditional Chinese Herbal Medicine: Chaihu (Bupleuri radix) by Both High-Performance Liquid Chromatographic and High-Performance Thinlayer Chromatographic Fingerprint and Chemometic Analysis," Journal of Chromatography A, Vol. 1216, No. 11, 2009, pp. 2150-2155. http://dx.doi.org/10.1016/j.chroma.2008.10.127

[15] P. S. Xie and S. P. Li, “Chapter 2: Back to the Future in Quality Control of Chinese Herbal Medicines/Quality Control: Developments, Methods and Applications,” Nova Science Publishing Co. Ltd., 2013, pp. 47-68.

[16] M. Schwarz, B. Klier and H. Sievers, "Herbal Reference Standards,” Planta Medica, No. 75, 2009, pp. 689-703.

[17] K. Helliwell, "Herbal reference Standards (Reader's Tribute),” Pharmeuropa, Vol. 18, No. 2, 2006, pp. 235-238.

\section{Abbreviations}

ChP = Pharmacopoeia of the Peoples Republic of China

$\mathrm{CHM}=$ Chinese Herbal Medicine

TCM = Traditional Chinese Medicine

CRS $=$ Chemical Reference Substance

HRS $=$ Herbal Reference Substance

ERS $=$ Extractive Reference Substance

USP $=$ The United States Pharmacopoeia

$\mathrm{EuP}=$ The European Pharmacopoeia 